\title{
Overutilization of Breast Cancer Screening in the US: Awareness of a Growing Problem
}

\author{
Mara A. Schonberg, MD, MPH \\ Division of General Medicine and Primary Care, Department of Medicine, Harvard Medical School, Beth Israel Deaconess Medical Center, Boston, \\ MA, USA.
}

J Gen Intern Med 33(3):238-40

DOI: $10.1007 / \mathrm{s} 11606-017-4258-9$

(c) Society of General Internal Medicine 2017

$\mathrm{R}$ ecently, a 72-year-old female patient of mine with stage IV metastatic thyroid cancer came in for a routine visit, accompanied by her daughter. As we began to talk, the patient mentioned that the medical assistant (MA) who roomed her said that she needed a mammogram since she had not been screened in 2 years. Based on my patient's thyroid cancer and other medical problems, I estimated her life expectancy to be $<10$ years. I told her that I did not think that she needed a mammogram due to the low likelihood of benefit and because there are risks to being screened. The patient was comfortable not having a mammogram, but her daughter still wanted her to have it done, though she has not yet scheduled the test. Ironically, the MA who recommended the mammogram was doing so in the name of "quality." Receipt of biennial screening mammograms among women aged $50-74$ years is a national quality metric, and my practice receives a financial incentive if nearly all (90\%) women aged 50-74 are screened. ${ }^{1}$ To help achieve this goal, numerous systems are in place in our practice to ensure that as many women as possible are screened, including availability of same-day mammograms and training of MAs to identify and notify patients in need of screening. While these efforts result in more women being screened, they also lead to more screening in women who are unlikely to benefit, or low-value care. ${ }^{2}$

Underutilization of health care services, especially among underserved and underrepresented populations, has been a concern of health care professionals for years. However, increasingly there is recognition that overutilization (utilization of health care services among individuals very unlikely to benefit) is also an issue, especially regarding cancer screening. ${ }^{2}$ To maximize the benefits of screening while minimizing the harms, ideally, patients at higher risk with adequate life expectancy to benefit would be screened, while those at lower risk with little chance of benefit would not be screened, or at least would be directed towards less frequent and less invasive screening. However, in a health care system designed to maximize the use of cancer screening tests, particularly for

Published online December 20, 2017 breast cancer screening, achieving high-value care can be challenging.

Two articles in this issue identified women for whom breast cancer screening is being overutilized. ${ }^{3,4}$ The first, by Sadigh et al., examined use of mammography screening among 34,127 female Medicare beneficiaries aged 65-108 who were newly diagnosed with advanced lung or colorectal cancer between 2000 and 2011. They found that $9 \%$ of these women had a screening mammogram after diagnosis, despite the fact that advanced lung and colorectal cancer both have an overall 5 -year survival of less than $20 \%$. They also found that, similar to recall rates in women without advanced cancer, $11 \%$ of the women screened underwent subsequent diagnostic testing. The authors thought that this recall rate was high, as detecting an early breast cancer in these women was unlikely to help them live longer or better. They questioned whether a higher threshold for recall would be appropriate for women with shorter life expectancy.

The second article, by Hill et al., examined utilization of breast cancer screening with magnetic resonance imaging (MRI) among 348,955 women who underwent screening mammography between 2007 and 2014 at one of five regional breast imaging registries participating in the Breast Cancer Surveillance Consortium. Guidelines recommend screening breast MRI for women with high-risk gene mutations or with $>20$ $25 \%$ lifetime breast cancer risk as estimated by risk calculators based on family history (e.g., Claus or Tyrer-Cuzick). ${ }^{5}$ Since risk estimates from these risk calculators were not available, the authors used a history of 2 or more first-degree relatives with breast cancer as a proxy (risk models would generally estimate these women as having $>20 \%$ lifetime risk). The authors found that $94 \%$ of women with two or more first-degree relatives with breast cancer did not receive screening breast MRI, while $0.36 \%(1232 / 344,858)$ of women without two first-degree relatives with breast cancer were screened. While $0.36 \%$ is a low proportion, the authors noted that it implies that 140,000 US women are receiving screening MRI with little chance of benefit. While breast MRI has higher sensitivity than mammography for identifying breast cancers, it has lower specificity. ${ }^{6}$ Therefore, the use of breast MRI in women at low or average risk leads to more women experiencing false-positive tests, and may result in more women being overdiagnosed and overtreated for breast cancer. In addition, breast MRI screening requires the use of intravenous gadolinium contrast. 
These articles suggest that changes are needed in order to increase the value of breast cancer screening in the US. First, guidelines should be written as clearly as possible and should be cognizant of indication creep ${ }^{7}$ (diffusion of health care services to populations for whom the service has not been tested) and thoughtful about the settings in which the guidelines are likely to be implemented. Guidelines should specify the populations for whom the guidelines do and do not apply. Currently, the US Preventive Services Task Force (USPSTF) recommends mammography screening for women 50-74 years of age, with no exceptions, and states that the evidence is insufficient to assess the benefits and harms of mammography screening in women $>74$ years of age; other organizations recommend not screening women with $<10$ year life expectancy. ${ }^{8,9}$ Guidelines based on value recommend against screening women $>74$ years of age at average risk and against screening women with $<10$-year life expectancy. $^{2}$ Greater specificity would help facilitate implementation of these guidelines. For example, guidelines should indicate what risk threshold and which risk calculators should be used to categorize older women as being at higher risk for breast cancer. Similarly, guidelines could indicate how we should decide whether a patient has $<10$-year life expectancy. Are mortality indices on ePrognosis.org sufficient? If so, which risk threshold for 10 -year mortality $(50 \%$ risk of 10-year mortality? $60 \%$ risk?) is appropriate to recommend stopping screening?

Furthermore, when data are insufficient for a guideline organization to make a recommendation for a specific population, organizations might state the optimal default action. Currently, some primary care physicians (PCPs) tend to recommend screening for women aged $>75$ years, while others tend to recommend stopping screening at age 75 . Ideally, PCPs should be encouraged to allow their patients to make informed decisions about whether to be screened, based on a realistic understanding of the potential benefits and harms and based on the patient's values and preferences. However, PCPs find it challenging to communicate the risks of screening, and both PCPs and patients find the concepts of lag-time to benefit and overdiagnosis difficult to comprehend. Therefore, PCPs need strategies on how to communicate these concepts, and need tools to individualize the potential benefits and harms of screening based on women's risk factors and life expectancy. In addition, once a patient is diagnosed with an advanced cancer, they are often seen by their oncologist more than their PCP. Oncology professional societies might suggest that oncologists discuss stopping breast cancer screening with women diagnosed with advanced lung or colorectal cancer. We must also recognize that patients and doctors may not wish to acknowledge or discuss poor prognosis as the rationale for stopping screening.

Guidelines on breast MRI screening are also challenging to follow. Lifetime risk of breast cancer varies greatly depending on which breast cancer risk calculator is used, and it is not clear that it is reasonable to use the same risk threshold to recommend breast MRI for all calculators. ${ }^{10}$ Also, risk calculators based on family history tend to take more time to use than is readily available to busy PCPs. If breast MRI screening is valuable, then guidelines should recommend practical ways for PCPs to identify women at high risk who may benefit. Based on Hill et al.'s study, some PCPs may already be using the Gail model to identify women who may benefit from breast MRI screening, since the authors found that women without a family history of breast cancer but with $>20 \%$ lifetime risk based on the Gail model were significantly more likely to be screened with breast MRI.

While changing practice is tough, other countries are beginning to take on the challenge of reducing overutilization of breast cancer screening. For example, France is completely reforming their approach to breast cancer screening to emphasize individualized risk and informed decision making. ${ }^{11}$ Ideally, here in the US, as we aim to improve the quality and value of care, we will design a system that allows women the opportunity to make an informed decision about whether to be screened, regardless of age. To do so will likely require that quality metrics shift from focusing on receipt of breast cancer screening to receipt of individualized and understandable information on the benefits and harms of breast cancer screening. In such a system, my hospital would not be financially penalized when my 72-year-old patient with metastatic thyroid cancer chooses not to be screened.

Corresponding Author: Mara A. Schonberg, MD, MPH; Division of General Medicine and Primary Care, Department of Medicine, Harvard Medical School Beth Israel Deaconess Medical Center, Boston, MA, USA (e-mail: mschonbe@bidmc.harvard.edu).

Funding source Dr. Schonberg is supported by an RO1 (CA181357) and R21 (CA212386) research grant from the National Cancer Institute (NCI), and by an RO1 (AG041869) fromthe National Institute on Aging (NIA).

\section{REFERENCES}

1. Breast Cancer Screening. Quality Positioning System (QPS). Measure Description Display Information. Available at: http://www.qualityforum. org/QPS/MeasureDetails.aspx?standardID=2372\&print=0\&entityTypeID=1 . Accessed November 9, 2017.

2. Harris RP, Wilt TJ, Gaseem A. A value framework for cancer screening: advice for high-value care from the American College of Physicians. Ann Intern Med 2015;162:712-7.

3. Sadigh G DR, Ward KC, Jiang R, et al. Downstream breast imaging in advanced cancer. $\mathrm{J}$ of Gen Intern Med. 2017. https://doi.org/10.1007/ s11606-017-4212-x.

4. Hill DA, Haas JS, Wellman R, et al. Utilization of breast cancer screening with magnetic resonance imaging in community practice. $\mathrm{J}$ of Gen Intern Med. 2017. https://doi.org/10.1007/s11606-017-4224-6.

5. Saslow D, Boetes C, Burke W, et al. American Cancer Society guidelines for breast screening with MRI as an adjunct to mammography. CA Cancer J Clin. 2007;57:75-89.

6. Bluemke DA, Gatsonis CA, Chen MH, et al. Magnetic resonance imaging of the breast prior to biopsy. JAMA. 2004;292:2735-42.

7. Riggs KR, Ubel PA. The role of professional societies in limiting indication creep. J Gen Intern Med. 2015;30:249-52. 
8. Siu AL. Screening for Breast Cancer: U.S. Preventive Services Task Force Recommendation Statement. Ann Intern Med. 2016;164:279-96.

9. Oeffinger KC, Fontham ET, Etzioni R, et al. Breast Cancer Screening for Women at Average Risk: 2015 Guideline Update From the American Cancer Society. JAMA. 2015;314:1599-614.
10. Ozanne EM, Drohan B, Bosinoff $\mathbf{P}$, et al. Which risk model to use? Clinical implications of the ACS MRI screening guidelines. Cancer Epidemiol Biomarkers Prev. 2013;22:146-9.

11. Barratt A, Jorgensen KJ, Autier P. Reform of the National Screening Mammography Program in France. JAMA Intern Med. 2017. 